\title{
Atuação dos pais e responsáveis no cuidado da criança em Unidade de Terapia Intensiva
}

\author{
Claudirene Milagres Araújo1, Isabela Mie Takeshita1, Carolina \\ Sant'Anna Gusmão' ${ }^{2}$, Letícia Fernandes de Oliveira ${ }^{2}$, Carla de Paula \\ Silveira', Alessandra Silva Lima Jardim ${ }^{1}$
}

11Faculdade Ciências Médicas de Minas Gerais, Brasil | claudirene_milagres@hotmail.com; isabelamie@gmail.com; carlapaulasilveira@gmail.com; aleenf@gmail.com | https://orcid.org/0000-0003-0241-4445; https://orcid.org/0000-0002-1710-7555; https://orcid.org/0000-0003-3092-5218; https://orcid.org/0000-0003-0108-5002

${ }^{2}$ Instituto Materno Infantil de Minas Gerias S/A, Brasil | carolinasgusmao@hotmail.com; leticiafernandes.lf281@gmail.com | https://orcid.org/0000-0002-8700-3292; https://orcid.org/0000-0002-9078-8799

Resumo: A família é o primeiro alicerce de uma criança, imprescindível para seu crescimento e desenvolvimento adequados. Em diversos contextos a criança passa por momentos de estresse, como na doença e na hospitalização. Essa última pode levar à instabilidade emocional e trauma. Logo, neste processo, é normal a criança sentir medo e outros sentimentos desagradáveis, devido à falta de maturidade para assimilar seu estado de saúde e por estar em um ambiente desconhecido e afastado de seus familiares. É imprescindível que a equipe multidisciplinar desenvolva um cuidado baseado no compartilhamento efetivo de informações, no acesso irrestrito ao filho, na participação efetiva dos pais na tomada de decisões e no respeito mútuo. Objetivo: Compreender a percepção do enfermeiro acerca da atuação dos pais ou responsáveis da criança internada em Unidade de Terapia Intensiva Pediátrica. Métodos: Estudo descritivo de abordagem qualitativa, por meio de entrevistas online guiadas por roteiro semiestruturado. Foi utilizada a estratégia bola de neve, a amostragem por saturação teórica e os depoimentos foram analisados pelo referencial de Bardin. Resultados: Participaram 13 profissionais enfermeiros. As categorias temáticas elaboradas foram: Atuação dos pais ou responsáveis no apoio emocional e melhora clínica Execução e restrição do cuidado realizado pelos pais ou responsáveis. Conclusão: $O$ enfermeiro entende como positiva a permanência e a realização de cuidados pelos pais ou responsáveis dentro da Unidade de Terapia Intensiva Pediátrica, pois auxilia na melhora clínica e no apoio psicológico, além de reaproximar a família do cuidado do filho.

Palavras-chave: Cuidados Críticos; Cuidados de Enfermagem; Família.

\section{Parental or responsible operations in childcare in intensive care unit}

Abstract: The family is a child's first foundation, essential for proper growth and development. In different contexts, the child goes through moments of stress, such as illness and hospitalization, which can lead to emotional instability and trauma. Therefore, in this process, it's normal for children to feel fear and other unpleasant feelings, due to the lack of maturity to assimilate their health status and for being in an unknown environment and away from their family members. It's essential that the multidisciplinary team develop care based on the effective sharing of information, unrestricted access to the child, the effective participation of parents in decision-making and mutual respect. Objective: To understand the perception of nurses about the performance of the parents or guardians of the child hospitalized in Pediatric Intensive Care Unit. Methods: Descriptive study with a qualitative approach, through online interviews guided by a semi-structured script. The snowball strategy was used, sampling by theoretical saturation and the testimonies were analyzed using the Bardin framework. Results : 13 nurses participated. The thematic categories elaborated were: Performance of parents or guardians in emotional support and clinical improvement; Execution and restriction of care provided by parents or guardians. Conclusion: The nurses understand as positive the permanence and the performance of care by parents or guardians within the Pediatric Intensive Care Unit, as it helps in clinical improvement and psychological support, in addition to bringing the family closer to the care of the child.

Keywords: Critical Care; Nursing Care; Family. 


\section{Introdução}

A família é o primeiro alicerce da criança, imprescindível para seu crescimento físico e desenvolvimento psíquico adequados. Como primeira unidade do cuidado, os pais ou responsáveis atuam no aprendizado, estimulam sua educação cognitiva, social e emocional (Ramos, Lima, Leal, Prado, Oliveira, Souza, Figueiredo, \& Leite, 2016).

A criança passa por momentos de estresse no decorrer do seu desenvolvimento, como a doença e a hospitalização. Esta última pode levar à instabilidade emocional e trauma, levando a criança sentir medo e outros sentimentos desagradáveis que ocorrem pela falta de maturidade para assimilar seu estado de saúde, inserida em um ambiente desconhecido e afastado dos familiares (Farias, BärtschiGabatz, Milbrath, Schwartz, \& Freitag, 2018).

A internação da criança em uma Unidade de Terapia Intensiva Pediátrica (UTIP) configura um momento crítico para a família. A presença dos pais ou responsáveis junto à criança, legalizada pela Lei ํㅜㅅ‥257, garante permanência em acomodações em tempo integral em terapia intensiva neonatal e cuidados intermediários (Brasil, 2016).

A hospitalização pediátrica demanda afeto, respeito e discussão de cada procedimento a ser realizado. $O$ acolhimento e o diálogo favorecem o vínculo entre a equipe de enfermagem, criança e pais, (Farias, BärtschiGabatz, Milbrath, Schwartz, \& Freitag, 2018; Fayed, Cameron, Fraser, Cameron, Al-Harbi, Simpson, Wakim, Chiu, \& Choong, 2020) propiciando um cuidado humanizado e integral (Camponogara, Pinno, Dias, Bonfada, Belmonte, \& Loiola, 2018).

Crianças com acometimentos crônicos, condição funcional deficitária ou necessidade de aparato tecnológico sofrem mais reinternações (da Silva, \& Fonseca, 2019), sendo válido que profissionais de UTIP tenham sensibilidade e atuem proporcionando acolhimento e proteção, isto deixará memórias que facilitarão cada internação (Guerra, Chesani, \& Bossardi, 2019).

A equipe multidisciplinar deve ainda desenvolver cuidado baseado no compartilhamento de informações e participação efetiva dos pais na tomada de decisões. A abordagem fisiológica com análise de desfechos clínicos tais como diagnóstico, prognóstico e mortalidade, têm sido utilizada amplamente nas UTIPs há anos. Entretanto, não cobre adequadamente as nuances de um cuidado centrado na família e na criança que possui aspectos funcionais e emocionais que permeiam a qualidade de vida e reabilitação (DenisLarocque, Williams, ST-Sauveur, Ruddy, \& Rennick, 2017). Complementando, Sampaio, Zonta, Ferreira \& Okido (2017) reforçam que o cuidado centrado exige instrumentalização e empoderamento da família.

Os benefícios da presença familiar durante a internação da criança são relatados por profissionais de saúde que atuam em UTIPs, porém não destacam o valor da presença da família para a equipe assistencial (Silva, Souza, Cabeça, \& Melo, 2020).

Este estudo é relevante pois busca compreender a interação dos pais ou responsáveis junto à equipe de enfermagem. Tal compreensão poderá enriquecer as reflexões das equipes assistenciais no sentido da valorização da família e, mais importante, do convívio conjunto durante a internação.

Diante do exposto, este estudo foi direcionado pelo seguinte questionamento: qual a percepção do enfermeiro em relação à atuação dos pais ou responsáveis no cuidado do filho durante a internação hospitalar em UTIP? O enfermeiro percebe essa atuação como positiva ou negativa?

\section{Objetivo}

Compreender a percepção do enfermeiro acerca da atuação dos pais e responsáveis no cuidado do filho durante a internação hospitalar em uma Unidade de Terapia Intensiva Pediátrica. 


\section{Métodos}

Trata-se de uma pesquisa exploratória, descritiva, com abordagem qualitativa realizada entre dezessete de agosto e cinco de setembro de 2020, com treze enfermeiros que atuam em UTIP. O estudo qualitativo o objetivo de interpretar o fenômeno em observação, baseia-se na percepção e compreensão humana, compreendendo e descrevendo um fenômeno (Brandão, Ribeiro, \& Costa, 2018).

O estudo não foi vinculado a nenhuma instituição, os sujeitos participaram da pesquisa, independente da instituição ao qual estavam vinculados. Foram capturados pela técnica da Bola de Neve que consiste em uma amostragem não probabilística que utiliza cadeias de referência para localizar possíveis participantes (Naderifar, Goli, \& Ghaljaie, 2017; Kirchherr, \& Charles, 2018). Um entrevistado indica a participação de outro até o momento que o estudo atinge o "ponto de saturação", ou seja, os interrogados passam a repetir os mesmos conteúdos já relatados pelos entrevistados anteriores.

A primeira entrevista foi no centro de especialização em Terapia Intensiva Pediátrica de Belo Horizonte, que forneceu o contato de um dos seus alunos enfermeiros.

Os critérios de inclusão foram o tempo de atuação em UTIP de três anos e vinculação à instituição do momento da coleta por no mínimo dois anos. O entrevistado tinha um conhecimento da rotina do serviço, práticas assistenciais relacionadas à criança e permanência da família na unidade. Como critério de exclusão: profissionais com tempo de formação inferior a três anos e vínculo no atual local de trabalho menor que dois anos.

O roteiro de entrevista possuía perguntas semiestruturadas como: Fale sobre a permanência dos pais ou responsáveis dentro da UTIP que você trabalha. Quais orientações são repassadas aos pais ou responsáveis em relação ao cuidado que eles podem executar em suas crianças? Você considera importante a participação dos pais ou familiares no cuidado da sua criança internada na UTIP? Você percebe alguma mudança na recuperação das crianças quando a família atua junto com a enfermagem no cuidado?

As entrevistas foram agendadas individualmente de acordo com a disponibilidade dos profissionais, por vídeo chamada, após assinatura do Termo de Consentimento Livre e Esclarecido (TCLE). O participante fornecia um contato telefônico de outro possível entrevistado. Os pesquisadores realizavam a transcrição literal dos dados, atentando para a saturação que foi determinada por todos os pesquisadores em conjunto ao identificar a repetição dos dados.

Os pesquisadores responsáveis foram dois acadêmicos do curso de graduação em Enfermagem. O pré-teste foi aplicado em três indivíduos, verificando adequação do instrumento. Como não houve alterações no instrumento de coleta de dados, os depoimentos do pré-teste foram mantidos como parte do estudo. A privacidade e o anonimato dos participantes foram garantidos por meio da utilização de código alfanumérico, dessa maneira, cada enfermeiro participante era identificado pelas letras "ENF", seguido do número que especifica a ordem dos depoimentos, por exemplo; ENF1, ENF2 etc.

Foi utilizada a análise de conteúdo de Bardin que aplica um conjunto de técnicas que decompõem as comunicações, utiliza procedimentos sistemáticos e objetivos para descrever o conteúdo das informações e conhecer aquilo que está por trás das palavras. As etapas percorridas na análise de dados, incluíram a pré-análise, com organização do material e a sistematização de ideias por meio da leitura flutuante, seleção das falas, levantamento de hipóteses e construção de indicadores, foram respeitados os critérios de exaustividade, representatividade, homogeneidade e pertinência. Durante a exploração do material ocorreu a codificação, categorização e identificação das unidades de registro e de contexto, respeitando-se o critério de exclusividade. Por fim, o tratamento dos resultados, inferência e interpretação, etapa que demanda intuição, análise reflexiva e crítica (Bardin, 2011). 
O estudo seguiu orientações da Resolução no 510/2016 (Brasil, 2016) de pesquisas com seres humanos e foi aprovado pelo Comitê de Ética e Pesquisa da Faculdade Ciências Médicas de Minas Gerais, sob parecer no4.190.114. As limitações do estudo dizem respeito à sua natureza qualitativa, uma vez que não são permitidas generalizações e transferência de resultados relativos à atuação de enfermeiros em UTIPs em cenários com características diversas. Torna-se muito desejável que outras dimensões, a partir dos resultados apresentados, possam ser consideradas como, por exemplo, a percepção dos pais quanto ao cuidado prestado, das demais categorias profissionais e, mesmo, da família.

\section{Resultados}

Participaram da pesquisa 13 enfermeiros que atuam em terapia intensiva pediátrica e neonatal em Belo Horizonte e região metropolitana. O processo de análise de conteúdo latente resultou em duas categorias: "Atuação dos pais ou responsáveis no apoio emocional e na melhora clínica"; "Execução e restrição do cuidado realizado pelos pais ou responsáveis".

\subsection{Atuação dos Pais e Responsáveis no Apoio Emocional e na Melhora Clínica}

Ao serem questionados sobre a permanência dos pais ou responsáveis dentro da UTIP, os enfermeiros afirmaram que tal prática é positiva, pois eles atuam no apoio emocional e no melhor enfrentamento da doença pela criança.

"É importante quando os pais estão presentes durante a internação, eles dão apoio emocional, por causa do vínculo e da confiança existente entre eles. $A$ criança se sente mais segura e corajosa e enfrenta melhor o processo da doença [...]" (ENF7).

"Quando os pais ficam junto durante internação, a criança fica mais calma e chora menos [...]. Os pequenos se deparam com um ambiente novo e diferente, ficam estressados. A presença da família traz mais tranquilidade, noto que com o passar dos dias a internação fortalece o vínculo da família" (ENF 2).

"A interação da família no cuidado do filho possibilita uma recuperação mais rápida, eles evoluem melhor [...], recebem alta hospitalar mais rápido" (ENF8).

Foi mencionado por uma enfermeira a importância dos pais ou familiares no estímulo da criança para que ela possa ter um desenvolvimento neuropsicomotor adequado a sua idade, quando esta fica internada por um grande período na UTIP.

"Quando a criança pequena fica internada por um longo período sem a presença contínua dos pais ou responsáveis, percebo que fica com o desenvolvimento mais lento e menos interativa, por mais que a equipe manipule, brinque e estimule [...], é diferente da mãe ou do pai que estimula a criança a maior parte do tempo" (ENF9).

A presença e participação dos pais ou responsáveis nos procedimentos faz com que as crianças se sintam mais seguras e abertas para os procedimentos realizados pela equipe de enfermagem. 
"Quando os pais ajudam nos procedimentos que a enfermagem realiza, as crianças ficam mais tranquilas e quietinhas. Os pais colocam gorro, máscara e luva, ajudam a acalmar o filho, assim consigo fazer mais rápido e melhor" (ENF9).

"A criança se sente mais corajosa em relação aos procedimentos e mais aberta com a aproximação da enfermagem, assim fica mais fácil fazer punções, sondagens e administrar medicação" (ENF7).

\subsection{Execução e Restrição do Cuidado Realizado pelos Pais ou Responsáveis}

Os pais ou responsáveis são orientados na admissão dos seus filhos na UTIP, quanto às rotinas do setor, com vistas à gestão de riscos de queda e infecções.

"Sempre orientamos sobre o risco de queda [...], falamos para o acompanhante sempre subir a grade da cama ao sair de perto da criança. Sobre o risco de infecção, [...] a lavagem das mãos a todo momento, não manipular os dispositivos invasivos da criança, ao utilizar o celular passar álcool nas mãos antes e depois e não encostar em outros leitos [...]." (ENF9)

"Os pais participam da administração de medicamentos, não podem fazer sozinhos, a criança tem dispositivos venosos [...], falo também do risco de infecção, sobre não pegarem nos dispositivos, oriento tudo na admissão do paciente e reforço a todo momento" (ENF2).

Os pais e responsáveis podem realizar cuidados básicos, corriqueiros em casa.

"Nas crianças estáveis em ar ambiente, os pais podem realizar troca de fralda, auxiliar no banho, dar a mamadeira e passar a dieta por sonda, a criança ficará mais tranquila" (ENF3).

"Os pais realizam os cuidados de higiene, amamentam no seio ou mamadeira, observam e comunicam os diferentes tipos de choro, observa a respiração [...]" (ENF12).

Os enfermeiros enfatizam que os pais de crianças com doenças crônicas e de internação prolongada são mais aptos e habilidosos para realizar cuidados mais complexos.

"A família de crianças com gastrostomia e traqueostomia que retornam à internação na UTIP, podem manipular e administrar medicação pela sonda, aspirar secreção, pois são cuidados que eles já estão treinados em casa [...]" (ENF5).

“[...] pacientes com sondas entéricas ou gástricas que estão há muito tempo internados podem ter suas medicações e dieta administradas pelos pais com o acompanhamento da enfermagem" (ENF 6).

"Onde trabalho realizamos uma dupla checagem com os pais, de todas as medicações, exames e coletas de exames" (ENF 2).

Entretanto, em crianças graves e hemodinamicamente instáveis, os cuidados são realizados pela equipe de enfermagem.

"Se a criança estiver muito crítica, grave, os pais não poderão prestar quase nenhum cuidado, vai ficar sobre a nossa responsabilidade tudo, porque é manipulação mínima para manter a estabilidade da criança [...]" (ENF3).

"Nas crianças intubadas, sedadas, em suporte ventilatório, o pai apenas observa os cuidados da enfermagem, eles não executam" (ENF8).

Ademais, os enfermeiros revelam a importância da capacitação dos pais ou responsáveis durante a internação e no preparo para a alta hospitalar, principalmente quando esta criança portará dispositivos assistenciais. 
"Os pais precisam se apropriar do cuidado principalmente quando o filho recebe alta e vai para casa com dispositivos de assistência, com várias demandas. Eles devem estar treinados para conseguir lidar com a situação [...]" (ENF9).

"Quando a alta da criança é programada pelo médico, reforçamos a capacitação dos pais, que já é feita durante a internação, a família tem que ter tranquilidade em lidar com os dispositivos: sondas, traqueostomia e aspiração" (ENF2).

\section{Discussão}

Por Os cuidados realizados por pais e responsáveis na UTIP são percebidos como positivos e importantes no apoio emocional e na melhora clínica da criança. $\mathrm{O}$ acolhimento da criança e sua família se materializa por meio de um plano de cuidados individualizado que atenda valores e expectativas destes, resultando em uma assistência humanizada (Farias, BärtschiGabatz, Milbrath, Schwartz, \& Freitag, 2018; Valderrama Sanabria, \& Muñoz de Rodriguez, 2016).

Os depoimentos, em consonância com a Lei Federal n¹3.257, reforçam a permanência dos pais ou responsáveis junto a suas crianças durante a internação em UTIP (Brasil, 2016). Apresença e participação contribui para que as crianças se sintam mais seguras e abertas para o cuidado. A equipe de enfermagem deve demonstrar empatia pelos pais ou responsáveis, considerando sua presença como um direito, visto que traz como benefício a diminuição do estresse e propicia a colaboração da criança na internação (Junqueira, Leôncio, Vaz, Santos, Collet, \& Reichert, 2020).

O tratamento perpassa pelo estímulo diário da criança para que o seu desenvolvimento neuropsicomotor não seja interrompido na internação. A prática colaborativa de estímulos diários da criança deve ser realizada pela equipe multiprofissional e família, minimizando atrasos no desenvolvimento (Ribeiro, Borges, Balduino, Silva, \& Ramos, 2017).

Em relação à execução de cuidados, os pais são orientados sobre o que podem realizar junto aos seus filhos. O cuidado pode ser compartilhado entre a família e a equipe de enfermagem, desde que a criança apresente estabilidade hemodinâmica, respiratória e não esteja em uso de dispositivos invasivos. A família pode realizar cuidados básicos, tais como amamentação, troca de fraldas e acompanhamento da administração de medicações (Ramos, Lima, Leal, Prado, Oliveira, Souza, Figueiredo, \& Leite, 2016; Junqueira, Leôncio, Vaz, Santos, Collet, \& Reichert, 2020).

Em crianças com quadro clínico agudo, dependentes de dispositivos invasivos assistenciais, os cuidados realizados pelos pais devem ficar restritos, uma vez que o uso dessas tecnologias pode causar desconforto para o familiar. Em casos de hospitalização por doença crônica, os pais se sentem mais seguros, uma vez que essa manipulação de dispositivos é parte da rotina diária da família (Ramos, Lima, Leal, Prado, Oliveira, Souza, Figueiredo, \& Leite, 2016; Macedo, Cibreiros, Morais \& Vieira, 2017). Neste contexto, é comum haver insegurança e medo pelo futuro do filho doente, mas o enfrentamento pode melhorar mediante a inclusão efetiva do familiar no processo assistencial, até mesmo promover a redução de erros e a segurança do paciente (Cieslak, Martins, Berté, \& Makuch, 2021).

Ressalta-se que a participação da mãe no cuidado do filho minimiza o sentimento de culpa pelo seu adoecimento. Os pais mantêm o vínculo afetivo com a criança, se sentem ativos e participantes no tratamento. A autonomia conquistada gera empoderamento, aptidão e confiança para cuidar de seus filhos após a alta da UTIP (Sampaio, Zonta, Ferreira \& Okido; 2017). 
A equipe multiprofissional tem um papel importante no apoio e capacitação dos pais, sendo fundamental o fortalecimento dessa relação, por meio de comunicação efetiva, considerando aspectos emocionais e fisiológicos. Como resultado, um atendimento efetivo, seguro e de qualidade será alcançado (Richards, Starks, Connor, \& Doorenbos, 2018; Azevedo, Hemesayh, \& Oliveira, 2019).

\section{Conclusões}

O período de internação da criança é um processo complexo, ela é retirada do lar e inserida em local diferente. A abordagem qualitativa permitiu uma aproximação da experiência do enfermeiro com os pais de crianças internadas em UTIP, possibilitou concluir que o enfermeiro entende como positiva a permanência e a realização de cuidados pelos pais ou responsáveis dentro da UTIP, pois auxilia na melhora clínica, no apoio psicológico e reaproxima a família do cuidado do filho.

O enfermeiro acredita que o vínculo entre a equipe e pais ou responsáveis deve ser fortalecido. $O$ acolhimento dos acompanhantes melhora o relacionamento e torna o cuidado compartilhado entre enfermagem e a família mais seguro e assertivo, contribuindo de forma significativa para a qualidade da prática assistencial.

Ademais, desperta novos caminhos para qualificar o cuidado intensivo pediátrico, contribuindo para a construção de outros estudos qualitativos que explorem outras facetas assistenciais, como a própria visão da criança, ou mesmo, dos pais, no desempenho dos cuidados dentro da UTIP.

\section{Referências}

Azevedo, E. C., Hemesayh, T. P., \& Oliveira, V. Z. (2019). A internação de um filho em unidade de terapia intensiva pediátrica: narrativas maternas. Revista da SBPH, 22 (1), 172-194. http://pepsic.bvsalud.org/scielo.php?script=sci_arttext\&pid=s1516-08582019000100010

Brandão C., Ribeiro A., Costa A. P. (2018). Investigação qualitativa: em que ponto estamos? Ciência \& Saúde Coletiva, 23(1), 4. https://doi.org/10.1590/1413-81232018231.27992017

Brasil. (2016). Lei no 13.257, de 8 de março de 2016. Dispõe sobre as políticas públicas para a primeira infância e altera a Lei no 8.069, de 13 de julho de 1990 (Estatuto da Criança e do Adolescente). Diário Oficial da República Federativa do Brasil, Brasília. http://www.planalto.gov.br/ccivil_03/_Ato2015-2018/2016/Lei/L13257.htm

Brasil. (2016). Resolução nำ510, de 07 de abril de 2016. Dispõe sobre as normas aplicáveis a pesquisas em ciências humanas e sociais. Diário Oficial da República Federativa do Brasil, Brasília. https://www.in.gov.br/materia/-/asset_publisher/Kujrw0TZC2Mb/content/id/22917581

Bardin L. Análise de conteúdo. Lisboa: Edições 70; 2011

Camponogara, S., Pinno, C., Dias, G. L., Bonfada, M. S., Belmonte, T. D. J., \& Loiola, C. N. (2018). Feelings paid by parents of children hospitalized in intensive neonatal and pediatric therapy units/Sentimentos vivenciados por pais de crianças hospitalizadas em unidades de terapia intensiva neonatal e pediátrica/Sentimientos vivenciados por padres de niños. Revista de Enfermagem da UFPI, 7(4), 43-47. https://revistas.ufpi.br/index.php/reufpi/article/view/7109/pdf

Cieslak, G., de Lima Martins, T. C., Berté, M. Â., \& Makuch, D. M. V. (2021). Interfaces da presença familiar no tratamento da criança gravemente enferma. Revista Eletrônica Acervo Saúde, 13(2), e6314-e6314. https://acervomais.com.br/index.php/saude/article/view/6314/4131

Denis-Larocque, G., Williams, K., ST-Sauveur, I., Ruddy, M., \& Rennick, J. (2017). Nurses' perceptions of caring for parents of children with chronic medical complexity in the pediatric intensive care unit. Intensive and Critical Care Nursing Journal, 43(1), 149-155. https://doi.org/10.1016/j.iccn.2017.01.010 
Farias, D., BärtschiGabatz, R. I., Milbrath, V. M., Schwartz, E., \& Freitag, V. L. (2019). Percepção infantil sobre a necessidade de hospitalização para o reestabelecimento da saúde. Revista Enfermagem Atual In Derme, 87(25), 1-7. https://doi.org/10.31011/reaid-2019-v.87-n.25art.186

Fayed, N., Cameron, S., Fraser, D., Cameron, J. I., Al-Harbi, S., Simpson, R., Wakim, M., Chiu, L., \& Choong, K. (2020). Priority Outcomes in Critically III Children: A Patient and Parent Perspective. American Journal of Critical Care, 29 (5), e94-e103. https://doi.org/10.4037/ajcc2020188

Guerra, C. M., Chesani, F. H., \& Bossardi, C. N. (2019). UNIDADE DE TERAPIA INTENSIVA PEDIÁTRICA: VISÃO DA CRIANÇA SOBRE O PROCESSO DE INTERNAÇÃO. Revista Univap, 25(48), 176-188. https://revista.univap.br/index.php/revistaunivap/article/view/2205/1545

Junqueira, C. C. S., Leôncio, A. B. A., Vaz, E. M. C., Santos, N. C. C. B., Collet, N., \& Reichert, A P. (2020). Estimulação de crianças com síndrome congênita pelo zika vírus no domicílio: desafios do cuidador. Revista Gaúcha de Enfermagem, 41(1), 1-9. https://doi.org/10.1590/1983-1447.2020.20190247

Kirchherr, J., \& Charles, K. (2018). Enhancing the sample diversity of snowball samples: Recommendations from a research project on anti-dam movements in Southeast Asia. PloS one, 13(8), e0201710. https://doi.org/10.1371/journal.pone.0201710

Macedo, I. F., Souza, T. V., Oliveira, I. C. S., Cibreiros, A. S., Morais, R. C. M., \& Vieira, R. F. C. (2017). Nursing team's conceptions about the families of hospitalized children. Revista Brasileira de Enfermagem, 70(5), 904-911. https://doi.org/10.1590/0034-7167-2016-0233

Martins, P. L., Azevedo, C. S., \& Afonso, S. B.C. (2018). O papel da família nos planos de tratamento e no cuidado pediátrico hospitalar em condições crônicas complexas de saúde. Revista Saúde e Sociedade, 27(4), 1218-1229. https://doi.org/10.1590/S010412902018170402

Naderifar, M., Goli, H., \& Ghaljaie F. (2017). Snowball Sampling: A Purposeful Method of Sampling in Qualitative. Strides in Development of Medical Education Journal, 14(3), e67670. http://dx.doi.org/10.5812/sdme.67670

Ramos, D. Z., Lima, C. Z., Leal, A. L., Prado, P. F., Oliveira, V. V., Souza, A. A. M., Figueiredo, M. I., \& Leite, M. T. S. (2016). A participação da família no cuidado às crianças internadas em unidade de terapia intensiva. Revista Brasileira em Promoção da Saúde, 29(2), 189-196. https://doi.org/10.5020/18061230.2016.p189

Richards, C. A., Starks, H., Connor, M. R., \& Doorenbos, A. Z. (2018). Elements of FamilyCentered Care in the Pediatric Intensive Care Unit: An Integrative Review. Journal of Hospice \& Palliative Nursing, 19(3), 238-246. https://dx.doi.org/10.1097\%2FNJH.0000000000000335

Ribeiro, K. R. A., Borges, S. P., Balduino, J. A. S., Silva, F. A., \& Ramos, T. M. S. T. (2017). Dificuldades encontradas pela enfermagem para implementar a humanização na unidade de terapia intensiva. Revista de Enfermagem da Universidade Federal do Piauí, 6(2), 51-56. https://doi.org/10.26694/reufpi.v6i2.5777

Sampaio, A. A., Zonta, J. B., Ferreira, F. Y., \& Okido, A. C. C. (2017). Family-centered care in a pediatric intensive care unit: professionals' perceptions. Rene, 18(4), 515-520. https://doi.org/10.15253/2175-6783.2017000400013

da Silva, P. S. L., \& Fonseca, M. C. M. (2019). Which children account for repeated admissions within 1 year in a Brazilian pediatric intensive care unit? Jornal de Pediatria, 95(5), 559-566. https://doi.org/10.1016/j.jped.2018.04.009

Silva, C. C. D., Souza, M. A. D., Cabeça, L. P. F., \& Melo, L. D. L. (2020). Modos de ser de profissionais de enfermagem em terapia intensiva pediátrica: vivências com famílias. Revista Mineira de Enfermagem, 24, 1-8.

Valderrama Sanabria, M. L., \& Muñoz de Rodriguez, L. (2016). Needs of parents in caring for their children in a Pediatric Intensive Care Unit. Investigación y Educación en Enfermería, 34(1), 29-37. https://doi.org/10.17533/udea.iee.v34n1a04

Vaz, J. C., Milbrath, V. M., Gabatz, R. I. B., Krug, F. R., Hirschmann, B., \& Oliveira, M. M. (2018). Cuidado à família da criança com doença crônica. Revista de Enfermagem da Universidade Federal de Pernambuco, 12(5), 1397-1408. https://doi.org/10.5205/1981-8963v12i5a230852p1397-1408-2018 